PROCEEDINGS OF THE

AMERICAN MATHEMATICAL SOCIETY

Volume 129, Number 6, Pages 1805-1815

S 0002-9939(00)05699-9

Article electronically published on November 2, 2000

\title{
FIRST RETURN PROBABILITIES OF BIRTH AND DEATH CHAINS AND ASSOCIATED ORTHOGONAL POLYNOMIALS
}

\author{
HOLGER DETTE
}

\author{
(Communicated by Claudia M. Neuhauser)
}

\begin{abstract}
For a birth and death chain on the nonnegative integers, integral representations for first return probabilities are derived. While the integral representations for ordinary transition probabilities given by Karlin and McGregor (1959) involve a system of random walk polynomials and the corresponding measure of orthogonality, the formulas for the first return probabilities are based on the corresponding systems of associated orthogonal polynomials. Moreover, while the moments of the measure corresponding to the random walk polynomials give the ordinary return probabilities to the origin, the moments of the measure corresponding to the associated polynomials give the first return probabilities to the origin.

As a by-product we obtain a new characterization in terms of canonical moments for the measure of orthogonality corresponding to the first associated orthogonal polynomials. The results are illustrated by several examples.
\end{abstract}

\section{INTRODUCTION}

Let $\left(X_{n}\right)_{n \in \mathbb{N}_{0}}$ denote a birth and death chain on the nonnegative integers $\{0,1$, $2, \ldots\}$ and let $P$ govern $\left(X_{n}\right)_{n \in \mathbb{N}_{0}}$ with one-step upward transition probabilities $u_{i}$ (birth), one-step downward transition probabilities $d_{i}$ (death) and holding probabilities $h_{i}(i \geq 0)$, where $u_{i}>0, d_{i+1}>0$ and $u_{i}+d_{i}+h_{i} \leq 1(i \geq 0)$. The case $u_{i}+d_{i}+h_{i}<1(i \geq 0)$ corresponds to a permanent absorbing state $i^{*}$, which can only be reached from state $i$ with probability $1-u_{i}-d_{i}-h_{i}$. Let $P_{x}$ denote the corresponding probability for the process started at $X_{0}=x$. It was shown by Karlin and McGregor [12] that the $n$-step transition probabilities of the process can be represented as

$$
P_{i}\left(X_{n}=j\right)=\pi_{j} \int_{-1}^{1} t^{n} R_{i}(t) R_{j}(t) d \psi(t)
$$

where

$$
\pi_{j}=\frac{u_{0} \ldots u_{j-1}}{d_{1} \ldots d_{j}}=\left[\int_{-1}^{1} R_{j}^{2}(t) d \psi(t)\right]^{-1}
$$

Received by the editors April 8, 1999 and, in revised form, September 7, 1999.

1991 Mathematics Subject Classification. Primary 60J15; Secondary 33C45.

Key words and phrases. Birth and death chain, spectral measure, orthogonal polynomials, associated polynomials, canonical moments. 
$\left(j \geq 1 ; \pi_{0}=1\right), \psi$ is a probability measure on the interval $[-1,1]$, called spectral measure of $P$, and $R_{j}(t)$ is a polynomial of degree $j$ defined recursively by $R_{-1}(t)=$ $0, R_{0}(t)=1$,

$$
t R_{n}(t)=u_{n} R_{n+1}(t)+h_{n} R_{n}(t)+d_{n} R_{n-1}(t) \quad(n \geq 0) .
$$

The polynomials $R_{j}$ are called random walk polynomials (see Whitehurst [21], van Doorn and Schrijner [5] or Charris and Ismail [3]) and play a particular role in the analysis of random walks on the nonnegative integers. Note that (1.1) implies that the polynomials $R_{j}$ are orthogonal with respect to the spectral measure, i.e.

$$
\int_{-1}^{1} R_{i}(t) R_{j}(t) d \psi(t)=\delta_{i j} \pi_{j}^{-1}
$$

where $\delta_{i j}$ denotes Kronecker's symbol.

The natural meaning of the formula (1.1) is a spectral representation of a linear operator acting on an appropriate Hilbert space (see e.g. Kac [11] or Karlin and McGregor [12]). To be precise let $P$ denote the matrix of one-step transition probabilities and consider the Hilbert space $L^{2}(\pi)$ of all complex sequences $\left(f_{i}\right)_{i \in \mathbb{N}_{0}}$ such that $\sum_{i=0}^{\infty}\left|f_{i}\right|^{2} \pi_{i}$ is finite. The transformation $(P f)_{0}=h_{0} f_{0}+u_{0} f_{1}$,

$$
(P f)_{i}=d_{i} f_{i-1}+h_{i} f_{i}+u_{i} f_{i+1},
$$

induces in $L^{2}(\pi)$ a bounded self-adjoint linear operator, say $T$, with norm $\leq 1$. If $e_{0}=(1,0,0, \ldots)$ denotes the first unit vector in $L^{2}(\pi)$, it can be shown (see Karlin and McGregor [12]) that

$$
P_{i}\left(X_{n}=j\right)=\pi_{j}\left\langle T^{n} R_{i}(T) R_{j}(T) e_{0}, e_{0}\right\rangle
$$

where $\langle\cdot, \cdot\rangle$ denotes the corresponding inner product on $L^{2}(\pi)$. Consequently the representation (1.1) is obtained by defining $\psi(x)=\left\langle E_{x} e_{0}, e_{0}\right\rangle$ where $\left\{E_{x}\right\}$ denotes the spectral resolution of the self-adjoint operator $T$. Uniqueness of $\psi$ follows from the fact that for $i=j=0$ the identity (1.1) determines all moments of the spectral measure $\psi$.

The corresponding first associated orthogonal polynomials are defined by

$$
R_{j}^{(1)}(t)=\int_{-1}^{1} \frac{R_{j+1}(t)-R_{j+1}(z)}{t-z} d \psi(z) \quad(j \geq 0)
$$

and satisfy the recurrence relation $R_{-1}^{(1)}(t)=0, R_{0}^{(1)}(t)=1 / u_{0}$,

$$
t R_{j}^{(1)}(t)=u_{j+1} R_{j+1}^{(1)}(t)+h_{j+1} R_{j}^{(1)}(t)+d_{j+1} R_{j-1}^{(1)}(t) \quad(j \geq 0)
$$

(see Szegö [20]). Associated polynomials already appear in Stieltjes' [18] fundamental work and are very natural because they are the numerators for the convergents of certain continued fractions. More precisely, the generating function of the common return probabilities can be represented as the Stieltjes transform of the spectral measure $\psi$ with a corresponding continued fraction expansion, i.e.

$$
\begin{aligned}
\frac{1}{z} P_{00}\left(\frac{1}{z}\right) & =\sum_{n=0}^{\infty} P_{0}\left(X_{n}=0\right) z^{-n-1}=\frac{1}{z} \sum_{n=0}^{\infty} \int_{-1}^{1}\left(\frac{x}{z}\right)^{n} d \psi(x) \\
& =\int_{-1}^{1} \frac{d \psi(x)}{z-x}=\frac{1}{z-h_{0}}-\frac{u_{0} d_{1}}{z-h_{1}}-\frac{u_{1} d_{2}}{z-h_{2}}-\ldots
\end{aligned}
$$


(see Dette and Studden [4]). The continued fraction on the right-hand side converges for $|z|>1$. The random walk and the first associated orthogonal polynomials appear in the representation of the $n$th convergent, i.e.

$$
\frac{R_{n-1}^{(1)}(z)}{R_{n}(z)}=\frac{1}{\sqrt{z-h_{0}}}-\frac{u_{0} d_{1}}{\left\lceil z-h_{1}\right.}-\ldots-\frac{\left.u_{n-2} d_{n-1}\right\rfloor}{\left\lceil z-h_{n-1}\right.} .
$$

It follows from Favard's theorem (see Chihara [2]) and the arguments of Karlin and McGregor [12] that there exists a probability measure $\psi^{(1)}$ on the interval $[-1,1]$ such that the first associated polynomials are orthogonal with respect to a measure $\psi^{(1)}$, i.e.

$$
\int_{-1}^{1} R_{i}^{(1)}(t) R_{j}^{(1)}(t) d \psi^{(1)}(t)=\delta_{i j} \frac{1}{\pi_{i+1}} \frac{1}{u_{0} d_{1}} .
$$

Our main results show that the associated polynomials and the corresponding measure of orthogonality play a similar role for the analysis of first return probabilities to the origin as the random walk polynomials $R_{j}(t)$ and the spectral measure for the analysis of the ordinary transition probabilities.

Theorem 1.1. Let $T_{0}=\inf \left\{n>0 \mid X_{n}=0\right\}$ denote the time of the first return to the origin of the birth and death chain $\left(X_{n}\right)_{n \in \mathbb{N}_{0}}$. Then the first return probabilities to the origin can be represented as

$$
f_{x, 0}^{n}=P_{x}\left(T_{0}=n\right)=u_{0} d_{1} \int_{-1}^{1} t^{n-1} R_{x-1}^{(1)}(t) d \psi^{(1)}(t)
$$

whenever $x \geq 1, n \geq 1$. Moreover, if $n \geq 2$,

$$
f_{0,0}^{n}=P_{0}\left(T_{0}=n\right)=u_{0} d_{1} \int_{-1}^{1} t^{n-2} d \psi^{(1)}(t) .
$$

The $k$ th associated orthogonal polynomials defined by the recursive relation $R_{0}^{(k)}(t)=1 / u_{k-1}, R_{-1}^{(k)}(t)=0$,

$$
t R_{j}^{(k)}(t)=u_{j+k} R_{j+1}^{(k)}(t)+h_{j+k} R_{j}^{(k)}(t)+d_{j+k} R_{j-1}^{(k)}(t)
$$

and the corresponding probability measure $\psi^{(k)}$ of orthogonality play a similar role for the analysis of the first return probabilities $f_{x, y}^{n} ; x>y$.

Corollary 1.2. For all $n \geq 1, y \geq 0$ and $x>y$ we have for the time $T_{y}=$ $\inf \left\{n>0 \mid X_{n}=y\right\}$ of first return to the state $y$

$$
f_{x, y}^{n}=P_{x}\left(T_{y}=n\right)=u_{y} d_{y+1} \int_{-1}^{1} t^{n-1} R_{x-y-1}^{(y+1)}(t) d \psi^{(y+1)}(t)
$$

where $R_{j}^{(y+1)}(t)$ denotes the $(y+1)$ th associated orthogonal random walk polynomial and $\psi^{(y+1)}$ the corresponding probability measure of orthogonality.

Note that these results give an interesting and important characterization of the first return probabilities to the origin. While the moments of the spectral measure $\psi$ give the common return probabilities to the origin, Theorem 1.1 shows that the moments of the measures $u_{0} d_{1} \psi^{(1)}$ and $d_{1} \psi^{(1)}$ give the first return probabilities $f_{0,0}^{n}(n \geq 2)$ and $f_{1,0}^{n}(n \geq 1)$, respectively. Similarly, Corollary 1.2 shows that the return probabilities $f_{k, k-1}^{n}$ are obtained as the moments of the measure $d_{k} \psi^{(k)}$ corresponding to the $k$ th associated orthogonal polynomials. 
A proof of Theorem 1.1 is given in Section 2. The proofs are based on a new relation between the spectral measure $\psi$ corresponding to the random walk polynomials $R_{n}(t)$ and the measure $\psi^{(1)}$ corresponding to the first associated polynomials $R_{n}^{(1)}(t)$. More precisely, it is shown that $u_{0} d_{1} d \psi^{(1)}(t)=\left(1-t^{2}\right) d \tilde{\psi}(t)$ where $\tilde{\psi}$ is the dual of the spectral measure $\psi$ which is obtained from $\psi$ by applying the transformation $x \rightarrow 1-x$ to the canonical moments of the probability measure $\psi$.

\section{The SPECTRAL MEASURE OF THE FIRST ASSOCIATED ORTHOGONAL POLYNOMIALS AND THE PROOF OF THEOREM 1.1}

The proof of Theorem 1.1 requires some basic facts about the theory of canonical moments. For a more detailed treatment of this theory we refer to the work of Dette and Studden [4]. Some basic facts are mentioned here for the sake of completeness. Let $\xi$ denote a probability measure on the interval $[-1,1]$ with ordinary moments $c_{1}, c_{2}, \ldots$ For a given set of moments let $c_{i}^{+}$denote the maximum of the $i$ th moment $\int_{-1}^{1} x^{i} d \mu(x)$ over the set of all probability measures $\mu$ having the given moments $c_{1}, c_{2}, \ldots, c_{i-1}$. Similary let $c_{i}^{-}$denote the corresponding minimum value. The canonical moments are defined by

$$
p_{i}=\frac{c_{i}-c_{i}^{-}}{c_{i}^{+}-c_{i}^{-}}, \quad i=1,2, \ldots
$$

Note that $0 \leq p_{i} \leq 1$ and that the canonical moments are in one-to-one correspondence with the ordinary moments. For this reason a probability measure $\xi$ on the interval $[-1,1]$ is uniquely determined by its sequence of canonical moments. Moreover, every sequence with elements in $[0,1]$ determines a probability measure on the interval $[-1,1]$ in this sense. The canonical moments are left undefined whenever $c_{i}^{+}=c_{i}^{-}$. If $i_{0}$ is the first index for which this equality holds, then $0<p_{k}<1$ $\left(k=1, \ldots, i_{0}-2\right)$ and $p_{i_{0}-1}$ must have the value 0 or 1 . In this case $\xi$ has finite support (see Skibinsky [17]). If $c_{i}^{+}>c_{i}^{-}$for all $i \in \mathbb{N}$, we formally put $i_{0}=\infty$ and the canonical moments are defined for all $i \in \mathbb{N}$. The following theorem relates the spectral measures of the random walk polynomials and first associated polynomials in terms of canonical moments.

Theorem 2.1. Let $p_{1}, p_{2}, \ldots$ denote the canonical moments of the spectral measure $\psi$ in the representation (1.1), $q_{j}=1-p_{j}$ and define $\tilde{\psi}$ as the probability measure with canonical moments $q_{1}, q_{2}, q_{3}, \ldots$ Then the first associated polynomials are orthogonal with respect to the measure $\left(1-t^{2}\right) d \tilde{\psi}(t)$; more precisely the corresponding probability measure is given by

$$
d \psi^{(1)}(t)=\frac{1}{u_{0} d_{1}}\left(1-t^{2}\right) d \tilde{\psi}(t) .
$$

Proof. Define $\zeta_{j}=q_{j-1} p_{j}(j \geq 1) ; q_{0}=1, \zeta_{0}=0$, by Corollary 2.3.5 in Dette and Studden [4] the monic orthogonal polynomials with respect to the measures $d \psi(t)$ and $\left(1-t^{2}\right) d \tilde{\psi}(t)$ satisfy the recursive relation $\left[P_{0}(t)=1, P_{-1}(t)=0, P_{0}^{(1)}(t)=\right.$ $\left.1, P_{-1}^{(1)}(t)=0\right]$,

$$
\begin{aligned}
& P_{j+1}(t)=\left(t+1-2 \zeta_{2 j}-2 \zeta_{2 j+1}\right) P_{j}(t)-4 \zeta_{2 j-1} \zeta_{2 j} P_{j-1}(t) \\
& P_{j+1}^{(1)}(t)=\left(t+1-2 \zeta_{2 j+2}-2 \zeta_{2 j+3}\right) P_{j}^{(1)}(t)-4 \zeta_{2 j+1} \zeta_{2 j+2} P_{j-1}^{(1)}(t)
\end{aligned}
$$


respectively. Because the random walk polynomials $R_{j}(t)$ are also orthogonal with respect to the measure $d \psi(t)$, we have from the monic form of (1.3)

$$
\begin{aligned}
-1+2 \zeta_{2 j}+2 \zeta_{2 j+1} & =h_{j} \quad\left(0 \leq j<i_{0}\right), \\
4 \zeta_{2 j-1} \zeta_{2 j} & =u_{j-1} d_{j} \quad\left(1 \leq j<i_{0}\right) .
\end{aligned}
$$

Inserting these identities into (2.4) and comparing the resulting recursion with the monic form of 1.6 shows that the polynomials $P_{j}^{(1)}(t)$ are the monic version of the first associated polynomials $R_{j}^{(1)}(t)$, i.e.

$$
d \psi^{(1)}(t)=c \cdot\left(1-t^{2}\right) d \tilde{\psi}(t) .
$$

For the determination of the remaining constant we use Remark 2.3.7 in Dette and Studden [4] and obtain from (2.5)

$$
1=\int_{-1}^{1} d \psi^{(1)}(t)=c \cdot 4 \zeta_{1} \zeta_{2}=c u_{0} d_{1}
$$

which completes the proof of Theorem 2.1.

Remark 2.2. Note that Theorem 2.1 gives a new characterization of the measure of orthogonality corresponding to the first associated orthogonal polynomials, which is different from the representations commonly used in the literature (see e.g. Sherman [15] or Grosjean [9]). For example, it is shown in Van Assche 19] that the Stieltjes transform of the orthogonality measure corresponding to the first associated orthogonal polynomials is given by the equation

$$
\int \frac{d \psi^{(1)}(y)}{x-y} \int \frac{d \psi(y)}{x-y}=\frac{1}{u_{0} d_{1}} \int \frac{y-h_{0}}{x-y} d \psi(y)
$$

and $\psi^{(1)}$ could be determined from $\psi$ by the Stieltjes inversion formula. If the measure $\psi$ is supported on a compact interval, the measure $\psi^{(1)}$ can be alternatively characterized by simply switching the canonical moments of $\psi$ and an application of (2.2).

Proof of the identity (1.9) in Theorem 1.1. From the identity (2.6) and Theorem 2.1 we obtain for all $|z|<1$

$$
z^{2} \int_{-1}^{1} \frac{\left(1-t^{2}\right) d \tilde{\psi}(t)}{1-t z} \cdot \int_{-1}^{1} \frac{d \psi(t)}{1-t z}=-1+\left(1-h_{0} z\right) \int_{-1}^{1} \frac{d \psi(t)}{1-t z}
$$

or equivalently

$$
\begin{aligned}
\left\{1-h_{0} z-z^{2} \int_{-1}^{1} \frac{\left(1-t^{2}\right) d \tilde{\psi}(t)}{1-t z}\right\}^{-1} & =\int_{-1}^{1} \frac{d \psi(t)}{1-t z} \\
& =\sum_{n=0}^{\infty} P_{0}\left(X_{n}=0\right) z^{n}=P_{00}(z)
\end{aligned}
$$

where $P_{00}(z)$ denotes the generating function of the return probabilities to the origin and the second equality follows from the integral representation (1.1). If

$$
F_{00}(z)=\sum_{n=0}^{\infty} P_{0}\left(T_{0}=n\right) z^{n}=1-\frac{1}{P_{00}(z)}
$$


denotes the generating function of the first return probabilities to the origin, we obtain

$$
F_{00}(z)=h_{0} z+z^{2} \int_{-1}^{1} \frac{\left(1-t^{2}\right) d \tilde{\psi}(t)}{1-t z}
$$

and an expansion of the right-hand side yields for $n \geq 2$ the representation (1.9) in Theorem 1.1, i.e.

$$
f_{0,0}^{n}=P_{0}\left(T_{0}=n\right)=\int_{-1}^{1} t^{n-2}\left(1-t^{2}\right) d \tilde{\psi}(t)=u_{0} d_{1} \int_{-1}^{1} t^{n-2} d \psi^{(1)}(t) .
$$

Proof of the identity 1.8 in Theorem 1.1. The proof of the remaining identity now follows by induction. For $x=1$ we have from (1.9)

$$
f_{1,0}^{n}=\frac{1}{u_{0}} f_{00}^{n+1}=d_{1} \int_{-1}^{1} t^{n-1} d \psi^{(1)}(t)=u_{0} d_{1} \int_{-1}^{1} t^{n-1} R_{0}^{(1)}(t) d \psi^{(1)}(t) .
$$

Similary, we have from the orthogonality relation (1.7)

$$
\begin{aligned}
& f_{2,0}^{1}=0=u_{0} d_{1} \int_{-1}^{1} R_{1}^{(1)}(t) d \psi^{(1)}(t), \\
& f_{2,0}^{2}=d_{1} d_{2}=u_{0} d_{1} \int_{-1}^{1} t R_{1}^{(1)}(t) d \psi^{(1)}(t) .
\end{aligned}
$$

Conditioning on $X_{1}$ now implies

$$
\begin{aligned}
f_{2,0}^{n} & =\frac{1}{u_{1}}\left\{f_{1,0}^{n+1}-h_{1} f_{1,0}^{n}\right\} \\
& =\frac{u_{0} d_{1}}{u_{1}} \int_{-1}^{1} t^{n-1}\left(t-h_{1}\right) R_{0}^{(1)}(t) d \psi^{(1)}(t)=u_{0} d_{1} \int_{-1}^{1} t^{n-1} R_{1}^{(1)}(t) d \psi^{(1)}(t)
\end{aligned}
$$

where the last identity follows by the recursive relation (1.6). The general step for $x+1>2$ follows by exactly the same argument, i.e.

$$
\begin{aligned}
f_{x+1,0}^{n} & =\frac{1}{u_{x}}\left\{f_{x, 0}^{n+1}-d_{x} f_{x-1,0}^{n}-h_{x} f_{x, 0}^{n}\right\} \\
& =\frac{u_{0} d_{1}}{u_{x}} \int_{-1}^{1} t^{n-1}\left\{\left(t-h_{x}\right) R_{x-1}^{(1)}(t)-d_{x} R_{x-2}^{(1)}(t)\right\} d \psi^{(1)}(t) \\
& =u_{0} d_{1} \int_{-1}^{1} t^{n-1} R_{x}^{(1)}(t) d \psi^{(1)}(t) .
\end{aligned}
$$

This completes the proof of Theorem 1.1.

Proof of Corollary 1.2. Let $\tilde{P}^{y}$ govern the birth and death chain $\left(X_{n}\right)_{n \in \mathbb{N}_{0}}$ with one-step transition probabilities $\left(y \in \mathbb{N}_{0}\right)$

$$
\begin{aligned}
& \tilde{u}_{j}=u_{j+y}, \\
& \tilde{d}_{j}=d_{j+y}, \\
& \tilde{h}_{j}=h_{j+y},
\end{aligned}
$$


for birth, death and no transition, respectively. From Theorem 1.1 we have for $x>y, n \geq 1$

$$
f_{x, y}^{n}=P_{x}\left(T_{y}=n\right)=\tilde{P}_{x-y}^{y}\left(T_{0}=n\right)=\tilde{u}_{0} \tilde{d}_{1} \int_{-1}^{1} t^{n-1} \tilde{R}_{x-y-1}^{(1)}(t) d \tilde{\varphi}(t)
$$

where $\tilde{R}_{j}^{(1)}(t)$ are the first associated orthogonal polynomials defined by the recurrence relation $\tilde{R}_{-1}^{(1)}(t)=0, \tilde{R}_{0}^{(1)}(t)=1$,

$$
\begin{aligned}
t \tilde{R}_{j}^{(1)}(t) & =\tilde{u}_{j+1} \tilde{R}_{j+1}^{(1)}(t)+\tilde{h}_{j+1} \tilde{R}_{j}^{(1)}(t)+\tilde{d}_{j+1} \tilde{R}_{j-1}^{(1)}(t) \\
& =u_{j+y+1} \tilde{R}_{j+1}^{(1)}(t)+h_{j+y+1} \tilde{R}_{j}^{(1)}(t)+d_{j+1+y} \tilde{R}_{j-1}^{(1)}(t)
\end{aligned}
$$

and $\tilde{\varphi}$ is the corresponding probability measure of orthogonality. The assertion of the Corollary now follows from (2.8), (2.9) and the recursive relation for the $(y+1)$ th associated orthogonal polynomials.

\section{EXAMPLES}

In general the calculation of the associated orthogonal polynomials and the corresponding measure of orthogonality is difficult and can only be done in very special cases (see e.g. Nevai [14], Grosjean [8, 9]). The following examples demonstrate that in some cases the results of Sections 1 and 2 can be useful for the identification of associated orthogonal polynomials and for the calculation of first return probabilities. The examples illustrate some applications of Theorem 2.1 in the context of orthogonal polynomials and demonstrate potential applications in the context of birth and death chains.

Example 3.1. Let $\psi$ denote the Beta distribution on the interval $[-1,1]$ with parameters $-1<\alpha<0, \beta=-1-\alpha$, i.e.

$$
\frac{d \psi}{d x}=\frac{1}{B(\alpha,-1-\alpha)}(1-t)^{\alpha}(1+t)^{-1-\alpha}, \quad t \in(-1,1) .
$$

The corresponding orthogonal polynomials are proportional to the Jacobi polynomials $P_{n}^{(\alpha,-1-\alpha)}(x)$ and the canonical moments of $\psi$ are given by

$$
p_{2 j}=\frac{1}{2}, \quad p_{2 j-1}=\frac{j-1-\alpha}{2 j-1} \quad(j \geq 1)
$$

(see Skibinsky [16]). From Theorem 8.2.5 in Dette and Studden [4] it follows easily that $\psi$ is the spectral measure of a random walk if and only if $\alpha=-1 / 2$. This case yields $p_{j}=1 / 2(j \in \mathbb{N}), u_{0}=1, u_{j}=d_{j}=1 / 2(j \in \mathbb{N})$ and some probabilistic aspects of the corresponding birth and death chain will be discussed in Example 3.2 .

By Theorem 2.1 the measure corresponding to the first associated orthogonal polynomials is given by $d \psi^{(1)}(t)=\left(1-t^{2}\right) d \tilde{\psi}(t)$, where $\tilde{\psi}$ has canonical moments $\tilde{p}_{j}=1-p_{j}$, i.e.

$$
\tilde{p}_{2 j}=\frac{1}{2}, \quad \tilde{p}_{2 j-1}=\frac{j+\alpha}{2 j-1}
$$

The measure corresponding to the canonical moments $\tilde{p}_{j}$ is again a Beta distribution with density

$$
\frac{d \tilde{\psi}}{d x}=\frac{1}{B(-1-\alpha, \alpha)}(1-t)^{-1-\alpha}(1+t)^{\alpha}, \quad t \in(-1,1)
$$


(see [16]). Consequently $\psi^{(1)}$ has a density proportional to $(1-t)^{-\alpha}(1+t)^{1+\alpha}$ and the first associated Jacobi polynomials $P_{n}^{(\alpha,-1-\alpha)^{(1)}}(x)$ are proportional to the Jacobi polynomials $P_{n}^{(-\alpha, 1+\alpha)}(x)$. This relation was already found by Grosjean [9] using a special structure of the corresponding weight function and by Wimp [22] [formula (40)] as a special case of a representation for the $c$-th associated Jacobi polynomial in terms of generalized hypergeometric functions.

Example 3.2. A simple example of a birth and death chain is the symmetric random walk with reflecting barrier for which the transition probabilities are given by $u_{0}=1, u_{j}=d_{j}=\frac{1}{2}(j \geq 1)$ and $h_{j}=0(j \geq 0)$. It follows from Karlin and McGregor [12] that the corresponding spectral measure is the arcsine distribution on the interval $[-1,1]$ and the random walk polynomials are the Chebyshev polynomials of the first kind, i.e. $R_{n}(t)=T_{n}(t)=\cos (n \arccos t)$. It is well known (see e.g. Wimp 22]) that the corresponding associated orthogonal polynomials of order $y$ are given by the Chebyshev polynomials of the second kind, i.e. $R_{n}^{(1)}(t)=U_{n}(t)$,

$$
R_{n}^{(y)}(t)=2 U_{n}(t)=2 \frac{\sin [(n+1) \arccos t]}{\sin [\arccos t]} \quad(y \geq 2)
$$

with corresponding probability measure $d \psi^{(y)}(t)=\frac{2}{\pi} \sqrt{1-t^{2}} d t$. Now Corollary 1.2 implies for the first return probabilities $f_{x, y}^{n}=0$ if $x+y+n$ is odd $(x>y)$ and for $x+y+n$ even $(x>y)$ we obtain

$$
\begin{aligned}
f_{x, y}^{n} & =u_{y} d_{y+1} \int_{-1}^{1} t^{n-1} R_{x-y-1}^{(y+1)}(t) d \psi^{(y+1)}(t) \\
& =\frac{1}{\pi} \int_{-1}^{1} t^{n-1} U_{x-y-1}(t) \sqrt{1-t^{2}} d t \\
& =\frac{1}{\pi} \int_{0}^{\pi}\left\{\cos ^{n}(s) \cos ((x-y) s)-\cos ^{n-1}(s) \cos ((x-y+1) s)\right\} d s \\
& =\frac{1}{2^{n}}\left(\begin{array}{c}
n \\
\frac{n-x+y}{2}
\end{array}\right)-\frac{1}{2^{n-1}}\left(\begin{array}{c}
n-1 \\
\frac{n-x+y}{2}-1
\end{array}\right)=\frac{x-y}{n 2^{n}}\left(\begin{array}{c}
n \\
\frac{n-x+y}{2}
\end{array}\right)
\end{aligned}
$$

where the third equality follows from the trigonometric representation of the Chebyshev polynomials of the second kind and the last identity is a consequence of a standard formula (see Jolley [10, eq. 55) for trigonometric functions.

Example 3.3. Consider the polynomials defined by $P_{0}(x)=1, P_{-1}(x)=0$,

$$
P_{n}(x)=2^{1-n}\left[T_{n}(x)-a T_{n-2}(x)\right] \quad(n \geq 2)
$$

where $a \in(-1,1)$ and $T_{n}(x)=\cos [n \arccos x]$ denotes the Chebyshev polynomial of the first kind. Grin̆spun [7] obtained the three term recurrence relation

$$
P_{n}(x)=x P_{n-1}(x)-\lambda_{n} P_{n-2}(x) \quad(n \geq 2)
$$

where

$$
\lambda_{2}=\frac{1+a}{2} ; \lambda_{3}=\frac{1-a}{4} ; \lambda_{n}=\frac{1}{4} \quad(n \geq 4) .
$$

He also found the corresponding probability measure of orthogonality which is given by

$$
\frac{d \psi}{d x}=\frac{1}{\pi} \frac{1-a^{2}}{(1+a)^{2}-4 a x^{2}} \frac{1}{\sqrt{1-x^{2}}}, \quad x \in(-1,1)
$$


Comparing the recurrence relation (3.2) with the general form (2.3) we obtain for the canonical moments of the probability measure $\psi: p_{2 j-1}=\frac{1}{2}(j \geq 1)$

$$
p_{2}=\frac{1+a}{2} ; \quad p_{2 j}=\frac{1}{2} \quad(j \geq 2) .
$$

By Theorem 8.3.1 in Dette and Studden [4] there exists a unique birth and death chain corresponding to $\psi$ with transition probabilities given by $u_{0}=1, u_{j}=1-d_{j}=$ $(1-a) / 2(j \in \mathbb{N})$. The dual spectral measure $\tilde{\psi}$ belongs to the same class and is given by

$$
\frac{d \tilde{\psi}}{d x}=\frac{1}{\pi} \frac{1-a^{2}}{(1-a)^{2}+4 a x^{2}} \frac{1}{\sqrt{1-x^{2}}}, \quad x \in(-1,1) .
$$

By Theorem 2.1 the first associated orthogonal polynomials are orthogonal with respect to the probability measure

$$
\frac{d \psi^{(1)}}{d x}=\frac{2}{\pi(1+a)} \frac{1-a^{2}}{(1-a)^{2}+4 a x^{2}} \sqrt{1-x^{2}} .
$$

Now results of Geronimus [6] show that the monic version of the first associated polynomials corresponding to (3.1) is given by

$$
P_{n}^{(1)}(x)=2^{1-n}\left[T_{n}(x)+\frac{1+a}{2} U_{n-2}(x)\right]
$$

where $U_{n}(x)$ denotes the Chebyshev polynomial of the second kind.

Example 3.4. Consider a birth and death chain with reflecting barrier at the origin (i.e. $u_{0}=1$ ) and

$$
u_{j}=\frac{j}{2 j+1}, \quad d_{j}=\frac{j+1}{2 j+1} \quad(j \in \mathbb{N}) .
$$

The first associated orthogonal polynomial are given by the recursive relation $R_{0}^{(1)}(t)=t, R_{-1}^{(1)}(t)=0$,

$$
(2 j+3) t R_{j}^{(1)}(t)=(j+1) R_{j+1}^{(1)}(t)+(j+2) R_{j-1}^{(1)}(t) \quad(j \geq 0)
$$

and can be identified as ultraspherical polynomials, i.e.

$$
R_{j}^{(1)}(t)=C_{j}^{(3 / 2)}(t)=P_{j+1}^{\prime}(t)
$$

where $P_{j+1}(t)$ denotes the $(j+1)$ th Legendre polynomial orthogonal with respect to the Lebesgue measure (see Szegö 20, pp. 80-83). The corresponding probability measure of orthogonality has density $\frac{3}{4}\left(1-t^{2}\right) I_{[-1,1]}(t)$. By Theorem 1.1 the first return probabilities to the origin are obtained as $f_{x, 0}^{n}=0$ if $x+n$ is odd and as

$$
\begin{aligned}
f_{x, 0}^{n} & =\frac{1}{2} \int_{-1}^{1} P_{x}^{\prime}(t) t^{n-1}\left(1-t^{2}\right) d t \\
& =-\frac{1}{2} \int_{-1}^{1} P_{x}(t) t^{n-2}\left\{n-1-(n+1) t^{2}\right\} d t \\
& =\frac{1}{4} x(x+1) \frac{\Gamma\left(\frac{n}{2}\right) \Gamma\left(\frac{n+1}{2}\right)}{\Gamma\left(\frac{n-x}{2}+1\right) \Gamma\left(\frac{n+x+3}{2}\right)}
\end{aligned}
$$

if $x+n$ is even. Here the last equality follows by straightforward algebra using formulae (22.13.8) and (22.13.9) in Abramowitz and Stegun [1]. 
Example 3.5. Our final example illustrates how the results of Sections 1 and 2 can be used for the investigation of the long term behaviour of the first return probabilities of a random walk with a finite state space and reflecting barriers. Consider for $\lambda>0$ the birth and death chain with finite state space $\{0, \ldots, N\}$, with one step transition probabilities $u_{0}=1$,

$$
u_{j}=\frac{j}{2(j+\lambda)}=1-d_{j} \quad(1 \leq j<N)
$$

and $u_{N}=0, d_{N}=1$. We will show that the first return probabilities to the origin converge to 0 with a geometric rate bounded by

$$
f_{x, 0}^{n}=O\left(\left(\cos N_{\lambda}\right)^{n}\right)
$$

$(n+x$ even) where

$$
N_{\lambda}= \begin{cases}\frac{j_{\lambda-1 / 2}}{\left\{(N+\lambda)^{2}+\left(1-4 / \pi^{2}\right) \lambda(1-\lambda)\right\}^{1 / 2}} & \text { if } \quad 0<\lambda \leq 1, \\ \frac{j_{\lambda-1 / 2}}{\left\{(N+\lambda)^{2}+\lambda(1-\lambda) / 3\right\}^{1 / 2}} & \text { if } \quad \lambda>1,\end{cases}
$$

and $j_{\lambda-1 / 2}$ denotes the minimal positive zero of the Bessel function $J_{\lambda-1 / 2}(x)$. To this end note that by (2.5) the canonical moments of the spectral measure $\psi$ are given by $p_{2 j-1}=1 / 2(1 \leq j \leq N), p_{2 j}=d_{j}(1 \leq j \leq N-1)$ and $p_{2 N}=1$. Therefore the measure $\tilde{\psi}$ has canonical moments $\tilde{p}_{2 j-1}=1 / 2(1 \leq j \leq N), \tilde{p}_{2 N}=0$ and

$$
\tilde{p}_{2 j}=u_{j}=\frac{j}{2(j+\lambda)}, \quad 1 \leq j<N .
$$

By Theorem 2.5.1 and 4.3.1 of Dette and Studden 4] the support of $\tilde{\psi}$ is finite and given by

$$
\operatorname{supp}(\tilde{\psi})=\left\{x \mid C_{N}^{(\lambda)}(x)=0\right\}
$$

where $C_{k}^{(\alpha)}(x)$ denotes the $k$ th ultraspherical polynomial (see Szegö 20], p. 80). If $x_{N, \Lambda}$ denotes the largest zero of $C_{N}^{(\lambda)}(x)$, then Theorems 1.1 and 2.1 show that

$$
\left|f_{x, 0}^{n}\right| \leq \frac{1+2 \lambda}{2+2 \lambda} \int_{-1}^{1}\left|t^{n-1} R_{x-1}^{(1)}(t)\right|\left(1-t^{2}\right) d \tilde{\psi}(t)=O\left(x_{N, \lambda}^{n}\right) .
$$

Now the assertion (3.8) follows by an application of bounds for the zeros of ultraspherical polynomials (see e.g. Laforgia [13], p. 216).

\section{ACKNOWLEDGEMENTS}

The author would like to thank W. Van Assche for some help with the references and I. Gottschlich who typed this manuscript with considerable technical expertise. He is also grateful to an unknown referee whose constructive comments resulted in a substantial improvement of an earlier version of this paper.

\section{REFERENCES}

1. M. Abramowitz, I.A. Stegun (1964). Handbook of Mathematical Functions. Dover, New York. MR 29:4914

2. T.S. Chihara (1978). An Introduction to Orthogonal Polynomials. Gordon and Breach, N.Y. MR 58:1979

3. J. Charris, M.E.H. Ismail (1986). On sieved orthogonal polynomials II: Random walk polynomials. Can. J. Math. 38, 397-415. MR 87j:33014a 
4. H. Dette, W.J. Studden (1997). The Theory of Canonical Moments with Applications in Statistics, Probability and Analysis. Wiley, N.Y. MR 98k:60020

5. E.A. van Doorn, P. Schrijner (1993). Random walk polynomials and random walk measures. J. Comput. Appl. Math. 49, 289-296. [MR 95c:60062]

6. S. Geronimus (1930). On a set of polynomials. Ann. Math. 31, 681-686.

7. Z.S. Griňspun (1966). On a class of orthogonal polynomials (in Russian). Vestrik Leningradskogo Universiteta Seria Matematiki, Mekhaniki i Astronomii 21, 147-149. MR 34:6172

8. C.C. Grosjean (1985). Theory of recursive generation of systems of orthogonal polynomials: An illustrative example. J. Comput. Appl. Math. 12-13, 299-318. MR 86g:33015

9. C.C. Grosjean (1986). The weight functions, generating functions and miscellaneous properties of the sequences of orthogonal polynomials of the second kind associated with the Jacobi and the Gegenbauer polynomials. J. Comp. Appl. Math. 16, 259-307. MR 88b:33020

10. L.B.W. Jolley (1961). Summation of Series. Dover, N.Y. MR 24:B511

11. M. Kac (1947). Random walk and the theory of Brownian motion. Amer. Math. Monthly 54, 369-391. MR 9:46c

12. S. Karlin, J. McGregor (1959). Random walks. Illinois J. Math. 3, 66-81. MR 20:7352

13. A. Laforgia (1980). Sugli zeri delle funzioni di Bessel. Calcolo 17, 211-220. MR 82i:33009

14. P. Nevai (1984). A new class of orthogonal polynomials. Proc. Amer. Math. Soc. 91, 409-415. MR 85f:42036

15. J. Sherman (1933). On the numerators of the convergents of the Stieltjes continued fractions. Trans. Amer. Math. Soc. 35, 64-87.

16. M. Skibinsky (1969). Some striking properties of Binomial and Beta moments. Ann. Math. Statist. 40, 1753-1764. MR 40:8106

17. M. Skibinsky (1986). Principal representations and canonical moment sequences for distributions on an interval. J. Math. Anal. Appl., 120:95-120, 1986. [MR 88c:60049]

18. T.J. Stieltjes (1894). Recherches sur les fractiones continues. Ann. Fac. Sci. Toulouse 8, J1-122. Ann. Fac. Scie Toulouse 9, A1-47.

19. W. Van Assche (1991). Orthogonal polynomials, associated polynomials and functions of the second kind. J. Comput. Appl. Math. 37, 237-249. MR 92j:33024

20. G. Szegö (1975). Orthogonal Polynomials. Amer. Math. Soc. Colloqu. Publ., Vol. 23, Providence, RI. MR 51:8724

21. T.A. Whitehurst (1982). An application of orthogonal polynomials to random walks. Pacific J. Math. 99, 205-213. MR 83m:60096

22. J. Wimp (1987). Explicit formulas for the associated Jacobi polynomials and some applications. Can. J. Math. 39, 983-1000. MR 88k:33023

Ruhr-Universität Bochum, Fakultät für Mathematik, 44780 Bochum, Germany

E-mail address: holger.dette@ruhr-uni-bochum.de 